\title{
Association between body mass index and hepatitis B antibody seropositivity in children
}

Yoowon Kwon, MD, Su Jin Jeong, MD, PhD

Department of Pediatrics, CHA Bundang Medical Center, CHA University School of Medicine, Seongnam, Korea

Background: The seropositivity rate of hepatitis B surface antigen (anti-HBs) antibodies is known to be $\geq 95 \%$ after hepatitis B virus vaccination during infancy. However, a low level or absence of anti-HBs in healthy children is discovered in many cases. Recent studies in adults reported that a reduced anti-HBs production rate is related to obesity.

Purpose: To investigate whether body mass index (BMI) affects anti-HBs levels in healthy children following 3 serial dose vaccinations in infancy.

Methods: We recruited 1,200 healthy volunteers aged 3, 5, 7, or 10 years from 4-day care centers and 4 elementary schools. All subjects completed a questionnaire including body weight, height, and vaccine type received. Levels of serum hepatitis B surface antigen (HBsAg) and anti-HBs in all subjects were analyzed using electrochemiluminescence immunoassay. The standardized scores (zscore) for each sex and age were obtained using the lambda-mu-sigma method in the 2017 Korean National Growth Charts for children and adolescents.

Results: Our subjects $(n=1,200)$ comprised 750 males $(62.5 \%)$ and 450 females $(37.5 \%)$. The overall anti-HBs seropositivity rate was $57.9 \%$ (695 of 1,200). We identified significant differences in mean BMI values between seronegative and seropositive groups (17.45 vs. 16.62, respectively; $P<0.001$ ). The anti-HBs titer was significantly decreased as the BMI $z$ score increased adjusting for age and sex ( $B=-15.725$; standard error $=5.494 ; P=0.004)$. The probability of anti-HBs seropositivity based on BMI $z$ score was decreased to an $\mathrm{OR}$ of 0.820 after the control for confounding variables $(95 \%$ confidence interval, $0.728-0.923 ; P=0.001)$.

Conclusion: There was a significant association between anti-HBs titer and BMI $z$ score after adjustment for age and sex. Our results indicate that BMI is a potential factor affecting anti-HBs titer in healthy children.

Key words: Anti-HBs, Hepatitis B, Seropositivity rate, BMI zscore, Child

\section{Key message}

Question: This study aimed to evaluate the association between BMI and anti-HBs level in healthy children following 3 serial dose vaccination in infancy.

Finding: After adjusting for age and sex, there was a significant association between anti-HBs titer and BMI zscore.

Meaning: Our findings revealed that BMI may be a factor potentially affecting anti-HBs titer so that booster vaccination or range of vaccination dose based on BMI and age can be considered.

\section{Introduction}

Hepatitis B virus (HBV) is a leading cause of acute or chronic hepatitis, and HBV infection continues to be a serious public health problem due to its high prevalence and association
Corresponding author: Su Jin Jeong, MD, PhD Department of Pediatrics, CHA Bundang Medical Center, CHA University School of Medicine, 59 Yatap-ro, Bundang-gu, Seongnam 13496, Korea Tel: +82-31-780-5349

Fax: +82-31-780-5011 E-mail: jinped@cha.ac.kr https://orcid.org/0000-0002-7388-8368

Received: 22 May, 2019

Revised: 27 July, 2019

Accepted: 9 August, 2019
Copyright (c) 2019 by The Korean Pediatric Society

This is an open-access article distributed under the terms of the Creative Commons Attribution NonCommercial License (http://creativecommons.org/ licenses/by-nc/4.0/) which permits unrestricted noncommercial use, distribution, and reproduction in any medium, provided the original work is properly cited. 
with chronic liver disease including cirrhosis and hepatocellular carcinoma. Vaccination is important because no complete cure for HBV infection exists, other than conservative treatment. ${ }^{1)}$ With the availability of low-cost, unrestricted production of recombinant vaccines, vaccination in all age groups, including newborns, is expected to eradicate hepatitis B infection. ${ }^{2)}$ In Korea, the hepatitis $B$ vaccine has finally been included in the National Mandatory Vaccination program since 1995 and the vaccination rate has reached $95 \% .{ }^{3)}$ In the 1980s, the overall positive rate of hepatitis B surface antigen (HBsAg) in Korea was approximately 7.25\%, which decreased to 4.38\% in 2001 and 3.7\% in 2005 after vaccination was initiated. ${ }^{4)}$ In a study conducted in 2008, the overall HBsAg seropositivity rate was found to have decreased to $2 \% .^{5}$ )

After the hepatitis B vaccine was included in the National Expanded Program on Immunization in Korea, all infants have been eligible for the basic course of HBV immunization, including 3 consecutive inoculations at 0,1 , and 6 months after birth. $\left.{ }^{6}\right)$ The preventive effect of the hepatitis $B$ vaccine as protection against infection is defined on the basis of the concentration of antibodies against hepatitis B surface antigen (anti-HBs) being $\geq 10 \mathrm{mIU} / \mathrm{mL}$. The anti-HBs seropositivity rate is known to be $\geq 95 \%$ after 3 serial doses of HBV vaccination during infancy. However, 5\%-15\% of normal adults are reported to fail to produce anti-HBs after 3 inoculations, and there have been a few cases of HBV infection in such nonresponders, although with mild clinical courses.")

Responses to hepatitis B vaccine vary among vaccine responders and vaccine-induced anti-HBs levels may progressively decrease, as shown in several studies. ${ }^{7-10)}$ The factors associated with immunogenicity of the hepatitis B vaccine in healthy individuals include the number of injections, site of injection, type of vaccine, use of adjuvant materials, and storage conditions of vaccines. ${ }^{11)}$ It is also known that characteristics and health status of subjects such as immunosuppression, hemodialysis, female gender, older age, obesity, smoking, and drinking are associated with decreased antibody formation. In particular, there have been studies in adults showing that a reduction in the rate of anti-HBs production is related to obesity. ${ }^{1,12-14)}$ However, there is a lack of studies addressing antiHBs production or retention in obese children after $\mathrm{HBV}$ vaccination in Korea, even though the obesity rate is gradually increasing among the population, especially in children and adolescents.

The present study investigated whether BMI affects anti-HBs seropositivity in healthy children who received 3 serial doses of vaccination in infancy.

\section{Methods}

\section{Subjects}

We recruited healthy volunteers aged 3 (36-47 months), 5 (60-71 months), 7 (84-95 months), or 10 years old (120-131 months), with 300 in each group, from 4-day care centers and 4 elementary schools in Seongnam-si, Gyeonggi-do at 2016. All 1,200 subjects had received 3 serial doses of recombinant hepatitis B vaccine at birth, at one month of age, and at 6 months of age.

Exclusion criteria in the study were HBV-positive parents or a family history of hepatitis B infection; a history of hepatitis B infection; additional $\mathrm{HBV}$ booster vaccination other than the standard vaccination; HBsAg positivity; receiving blood transfusion; or immunocompromised status including congenital or acquired immune disorder, hemodialysis, liver dysfunction, or cancer.

\section{Questionnaires and vaccination information}

All parents of the subjects completed a questionnaire about their children's age, sex, medical history, body weight, height, and type of vaccine. In most cases, information about vaccine type was obtained from the National Immunization Program website of the Korea Centers for Disease Control.

\section{Measurement of serum titer}

Serum HBsAg and anti-HBs in all subjects were analyzed via electrochemiluminescence immunoassay using the Roche Cobas 8000 (Roche Diagnostics, Indianapolis, IN, USA).

The cutoff index value of HBsAg was 1.0. Samples were considered positive if the $\mathrm{HBsAg}$ level was $>1.0$, and negative if it was $<1.0$. The measurement range of anti-HBs was $2-1,000 \mathrm{mIU} / \mathrm{mL}$, and seropositivity was defined as anti-HBs $\geq 10 \mathrm{mIU} / \mathrm{mL}$. Samples with anti-HBs $\geq 1,000 \mathrm{mIU} / \mathrm{mL}$ were recorded as $1,000 \mathrm{mIU} / \mathrm{mL}$ and samples below the detection limit $(2 \mathrm{mIU} / \mathrm{mL})$ were recorded as undetectable.

\section{BMl zscore}

We calculated the BMI of each subject based on their height and weight obtained via questionnaires. The standardized scores ( $z$ score) for sex and age were obtained using the LMS method in the 2017 Korean National Growth Charts for children and adolescents. ${ }^{15}$

\section{Statistical analysis}

All quantitative variables are presented as mean \pm standard deviation, and all qualitative variables are presented as percentage and number. Independent variables were analyzed using the chi-square test. T-test, Kruskall-Wallis test, or Mann-Whitney $U$ test were used to compare mean values of independent variables. Multiple and logistic regression analyses were used to calculate the odds ratio (OR) and 95\% confidence interval (CI), or the beta coefficients (B) and standard error (SE), after adjustment for age and sex. Statistical analysis was performed using the IBM SPSS Statistics ver. 23.0 (IBM Co., Armonk, NY, USA) and statistical significance was defined as $P \leq 0.05$. 


\section{Ethical considerations}

Written informed consent was obtained from the parents of all children or children enrolled in the study. The study was approved by the Institutional Review Board of CHA University (CHAIRB No.2015-196).

\section{Results}

\section{Subject characteristics}

A total of 1,200 subjects, 300 in each of the 4 groups of 3, 5, 7, or 10-year-olds, participated in the study. Of these, 750 were males (62.5\%) and 450 were females (37.5\%). The mean BMI value in each age group corresponded to the 50th percentile in each group. Euvax (recombinant DNA vaccine, LG Chemistry, Seoul, Korea) was the most commonly administered vaccine in all the groups (Table 1).

\section{Anti-HBs seropositivity rate based on age group}

The overall anti-HBs seropositivity rate in the subjects was 57.9\% (695 of 1,200). In the 3-year-old group, the anti-HBs seropositivity rate was 79.7\% (239 of 300). The seropositivity rates were 55.7\% (167 of 300), 51.7\% (155 of 300), and 44.7\% (134 of 300) in the 5, 7, and 10-year-old groups, respectively. As age increased, the anti-HBs seropositivity rate was significantly decreased $(P<0.001)$. There was no statistically significant difference in the anti-HBs seropositivity rate based on gender or type of vaccine in any of the age groups (Table 2).

Table 1. Baseline characteristics

\begin{tabular}{|c|c|c|c|c|c|}
\hline Characteristic & Total & 3-Year-old group & 5-Year-old group & 7-Year-old group & 10-Year-old group \\
\hline No. of patients & 1,200 & 300 & 300 & 300 & 300 \\
\hline Male sex & $750(62.5)$ & $216(72)$ & $189(63)$ & $193(64.3)$ & $152(50.7)$ \\
\hline \multicolumn{6}{|l|}{ Type of vaccine* $(n)$} \\
\hline Euvax Ba) & 577 & 130 & 148 & 156 & 143 \\
\hline Hepavax-gene $e^{\text {b) }}$ & 514 & 134 & 110 & 132 & 138 \\
\hline Hepamun ${ }^{c)}$ & 52 & 21 & 29 & 1 & 1 \\
\hline Others ${ }^{d)}$ & 57 & 15 & 13 & 11 & 18 \\
\hline Weight (kg) & & $16.07 \pm 2.00$ & $20.87 \pm 3.64$ & $26.88 \pm 5.30$ & $37.94 \pm 8.09$ \\
\hline Height (cm) & & $99.16 \pm 4.56$ & $113.54 \pm 5.49$ & $125.50 \pm 5.95$ & $142.52 \pm 8.91$ \\
\hline Body mass index $\left(\mathrm{kg} / \mathrm{m}^{2}\right)$ & & $16.29 \pm 1.16$ & $16.11 \pm 1.92$ & $16.95 \pm 2.33$ & $18.52 \pm 2.62$ \\
\hline
\end{tabular}

Values are presented as number (\%), number, or mean \pm standard deviation.

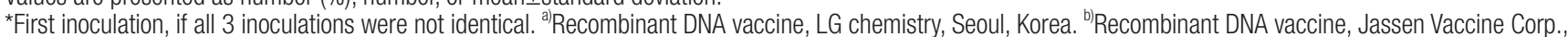
Seoul, Korea. ${ }^{\text {ch }}$ Recombinant DNA vaccine, SK Bio Science, Seongnam, Korea. ${ }^{d)}$ Heptis -BII, Hepa-B.

Table 2. Anti-HBs seropositivity rate based on age group

\begin{tabular}{|c|c|c|c|c|c|c|}
\hline & Titer $^{\text {a) }}<2$ & $2 \leq$ titer $<10$ & $10 \leq$ titer $<100$ & $100 \leq$ titer & Seropositivity rate (\%) & $P$ value \\
\hline Age & & & & & & $<0.001^{\text {b) }}$ \\
\hline 3 Years & 27 & 34 & 133 & 106 & 79.7 & $0.116^{c)}$ \\
\hline Male & 19 & 20 & 100 & 77 & & \\
\hline Female & 8 & 14 & 33 & 29 & & \\
\hline 5 Years & 97 & 36 & 107 & 60 & 55.7 & $0.667^{c)}$ \\
\hline Male & 57 & 25 & 68 & 39 & & \\
\hline Female & 40 & 11 & 39 & 21 & & \\
\hline 7 Years & 92 & 53 & 96 & 59 & 51.7 & $0.063^{c)}$ \\
\hline Male & 65 & 36 & 59 & 33 & & \\
\hline Female & 27 & 17 & 37 & 26 & & \\
\hline 10 Years & 108 & 58 & 91 & 43 & 44.7 & $0.366^{c)}$ \\
\hline Male & 64 & 24 & 42 & 22 & & \\
\hline Female & 44 & 34 & 49 & 21 & & \\
\hline
\end{tabular}

Values are presented as number of patients unless otherwise indicated.

Anti-HBs, antibody to hepatitis B surface antigen.

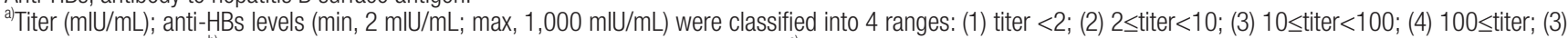
and (4), seropositive. ${ }^{\text {b) }} \mathrm{P}$ value for anti-HBs seropositivity rate based on age group. ${ }^{c} P$ value for anti-HBs seropositivity rate based on sex.

Boldface indicates a statistically significant difference with $P<0.05$. 


\section{Difference in mean BMI based on immunogenicity}

In all the subjects and in each age group, the mean BMI value in seronegative subjects tended to be higher than that in seropositive subjects. The mean BMI value in 505 seronegative subjects was $17.45 \pm 2.62$ and that in 695 seropositive subjects was $16.62 \pm 1.93$; this was a statistically significant difference $(P<0.001)$.

There was a tendency for differences in BMI values based on immunogenicity in all 4 age groups. In particular, BMI values in seronegative subjects were statistically significantly higher than those in seropositive subjects in the 7-year-olds (17.34 vs. 16.58, respectively; $P=0.005$ ) and the 10 -year-olds (18.85 vs. 18.11 , respectively; $P=0.016$ ) (Table 3).

\section{The association between $\mathrm{BMI} z$ score and the Anti-HBs titer}

We investigated the association between BMI $z$ score and antiHBs titer using multiple regression and logistic analyses. These indicated statistically significant associations of anti-HBs titer with BMI $Z$ score. The anti-HBs titer was decreased significantly as the BMI $Z$ score increased, adjusting for age and sex $(\mathrm{B}=-15.73, \mathrm{SE}=5.49, P=$ 0.004). In addition, the probability of anti-HBs seropositivity based on BMI $z$ score was decreased to an OR of 0.820 after controlling for confounding variables (95\% CI, 0.73-0.92; $P=0.001$ ) (Table 4).

\section{Anti-HBs titer in obese children}

Based on 2017 Korean National Growth Charts for children and adolescents, we defined obesity as BMI greater than 95th percentile

Table 3. Difference in mean body mass index based on immunogenicity in the entire group of subjects and in each age group $(n=1,200)$

\begin{tabular}{|c|c|c|}
\hline Anti-HBs titer & Body mass index $\left(\mathrm{kg} / \mathrm{m}^{2}\right)$ & $P$ value \\
\hline Total $(n=1,200)$ & & $<0.001$ \\
\hline Seronegative $^{a)}(n=505)$ & $17.45 \pm 2.62$ & \\
\hline Seropositive $^{\text {b) }}(n=695)$ & $16.62 \pm 1.93$ & \\
\hline 3-Year-old group $(n=300)$ & & 0.260 \\
\hline Seronegative $^{\text {a) }}(\mathrm{n}=61)$ & $16.44 \pm 1.60$ & \\
\hline Seropositive $^{b)}(n=239)$ & $16.25 \pm 1.02$ & \\
\hline 5 -Year-old group $(\mathrm{n}=300)$ & & 0.149 \\
\hline Seronegative $^{a)}(n=133)$ & $16.23 \pm 2.07$ & \\
\hline Seropositive $^{\text {b) }}(n=167)$ & $15.97 \pm 1.78$ & \\
\hline 7-Year-old group $(n=300)$ & & 0.005 \\
\hline Seronegative $^{a)}(n=145)$ & $17.34 \pm 2.37$ & \\
\hline Seropositive $^{\text {b) }}(n=155)$ & $16.58 \pm 2.24$ & \\
\hline 10-Year-old group $(n=300)$ & & 0.016 \\
\hline Seronegative $^{a)}(n=166)$ & $18.85 \pm 2.87$ & \\
\hline Seropositive $^{\text {b) }}(n=134)$ & $18.11 \pm 2.21$ & \\
\hline
\end{tabular}

Values are presented as mean \pm standard deviation.

Anti-HBs, antibody to hepatitis B surface antigen.

a) Anti-HBs titer $<10 \mathrm{mlU} / \mathrm{mL}$. b) $0 \mathrm{mlU} / \mathrm{mL} \leq$ anti-HBs titer.

Boldface indicates a statistically significant difference with $P<0.05$. in each sex and age group. ${ }^{15)}$ According to this definition, 132 children were obese among the total 1,200 children. The anti-HBs seropositivity rate in this obese group was $48.5 \%$ (64 of 132). The difference between the seropositivity rate in obese and nonobese group was more pronounced in older age groups, for instance, the seropositivity rate in obese and nonobese groups of 10-year-old age group were 15.8\% (3 of 19) and 46.6\% (131 of 281), respectively.

\section{Discussion}

The Advisory Committee on Immunization, World Health Organization, and the international group of hepatitis experts have defined the preventive effect of the hepatitis $B$ vaccine as the presence of anti-HBs concentration $\geq 10 \mathrm{mIU} / \mathrm{mL}$, representing protection against infection. ${ }^{7)}$ The rate of protective anti-HBs formation is known to be 16\%-40\% after the first inoculation and 80\%-95\% after the second inoculation. The third inoculation is performed as the booster, raising the antibody production rate to $98 \%-100 \% .{ }^{16)}$ However, there are many cases in which negative or low anti-HBs levels are found in healthy children. The highest concentration of anti-HBs level is known to be attained between 1 and 3 months after the last inoculation and subsequently, the anti-HBs level begins to decrease. The decrease in anti-HBs level is rapid after 1 to 2 years, but then slows down gradually. ${ }^{16)}$ In the present study, the seropositivity rate was found to be $79.7 \%$ at 3 years after vaccination, $57.7 \%$ after 5 years, $51.7 \%$ after 7 years, and $44.7 \%$ after 10 years. This is consistent with previous studies showing a statistically significant decrease in anti-HBs seropositivity rate with increasing age (Table 2).

It is known that the immune status of an individual is an important factor influencing antibody production. In addition, some studies have reported that several host factors in healthy individuals such as sex, age, obesity, smoking, and drinking are significantly related to immunogenicity of the hepatitis B vaccine. ${ }^{1)}$ In particular, obesity can be a notable risk factor for failure to produce anti-HBs, and it has been on the rise in both adults and children. In our study, we found significant differences in mean BMI values between seronegative and seropositive subjects (Table 3). Further, there was a significant relationship association between anti-HBs titer and BMI $z$ score with age and sex adjusted (Table 4). These are similar to results

Table 4. Multiple regression and logistic analyses of the association of BMl $z$ score with the anti-HBs titer $(n=1,200)$

\begin{tabular}{ccccccc}
\hline & \multicolumn{3}{c}{ Anti-HBs titer } & & \multicolumn{2}{c}{ Anti-HBs titer $^{\text {a) }}$} \\
\cline { 2 - 4 } \cline { 6 - 7 } & B & SE & $P$ value & & aOR $(95 \%$ Cl $)$ & $P$ value \\
\hline BMl z score & -15.73 & 5.49 & $0.004^{\text {b) }}$ & & $0.82(0.73-0.92)$ & 0.001 \\
\hline
\end{tabular}

BMI, body mass index; anti-HBs, antibody to hepatitis B surface antigen; $B$, beta; $\mathrm{SE}$, standard error; aOR, adjusted odds ratio; $\mathrm{Cl}$, confidence interval.

a) Seronegativity was defined as anti-HBs $<10 \mathrm{mlU} / \mathrm{mL}$, seropositivity was

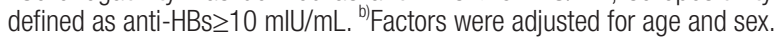


of previous studies in Iran, Turkey, China, and Belgium involving adults. ${ }^{11)}$ This finding indicates a trend of decreasing anti-HBs with increasing BMI, beyond the simple fact that obese individuals $\left(\mathrm{BMI} \geq 25 \mathrm{~kg} / \mathrm{m}^{2}\right.$ ) are significantly more likely to be nonresponders as reported in previous studies on adults. ${ }^{1,12,17)}$ In the present study as well, nonresponders accounted for 72\% (18 of 25) of the group with $\mathrm{BMI} \geq 23 \mathrm{~kg} / \mathrm{m}^{2}$ and 100\% (3 of 3) of the group with BMI $\geq 25 \mathrm{~kg} / \mathrm{m}^{2}$. Other previous studies on adults have reported vaccine deposition into gluteal fat as a risk factor among obese people. ${ }^{1,12,18-20)}$ In the present study, however, injection site was not found to be a risk factor associated with obesity because all subjects were injected in the thigh or the deltoid muscle during the neonatal period.

There have been several previous studies reporting poor vaccineinduced immune responses in obese individuals against influenza/ pH1N1, tetanus, or rabies, ${ }^{21-23)}$ and those reporting the effect of obesity on immune responses, ${ }^{24)}$ but the data were not of sufficient detail. ${ }^{25)}$ The mechanism via which obesity reduces antibody production is not clear, and it can be assumed that the relationship between obesity, inflammation, and vaccine immunogenicity is more complex than anticipated. It is currently a matter of discussion whether obesity should be regarded as a state of low-grade chronic inflammation which inhibits antibody production, ${ }^{26)}$ or whether dietary intake leading to obesity affects the gut leading to bacterial translocation and altered levels of immune activation. ${ }^{17)}$ Even if we cannot fully establish the mechanism currently, it is important to note that the proportion of obese individuals is increasing globally, particularly among adolescents.

Several studies have revealed that in individuals with the maximal anti-HBs level $\geq 10 \mathrm{mIU} / \mathrm{mL}$ after a 3 serial dose vaccination, the anamnestic response rapidly elevates the anti-HBs level to a protective level upon further exposure to the virus and maintains such immune system memory for at least 15 years. ${ }^{7,9,10)}$ However, there is no clear evidence that normal responders continue to have an anamnestic response for more than 15 years. Further, the immune system memory status in individuals is unknown unless the maximum antibody level after vaccination is tested, which is not done in most cases. ${ }^{7)}$ Due to the above reasons, there is no precise consensus on the necessity or scheduling of revaccination, and there have been no claims that booster doses are unnecessary. However, if obesity directly affects the immune system outcome, the possibility that the anamnestic response does not occur in obese nonresponders should be considered. Therefore, further studies on the anamnestic response in obese nonresponders are needed. In addition, booster vaccination based on BMI or a range of vaccination dose based on body weight can be considered.

This study is the first in Korea to report the association between BMI and anti-HBs seropositivity in healthy children. This study also has strengths in that it offers some considerations in the interpretation of anti-HBs in children.

The present study does have some limitations. First, the study was limited to the Gyeonggi area and may not represent all regions. Second, we could not evaluate whether the anti-HBs-negative subjects were nonresponders from the beginning, or were initial responders who lost anti-HBs production. Third, height and weight values were obtained via parental questionnaires, and the accuracy of the BMI may have been low. Fourth, because samples with anti$\mathrm{HBs} \geq 1,000 \mathrm{mIU} / \mathrm{mL}$ were recorded as $1,000 \mathrm{mIU} / \mathrm{mL}$, the results of anti-HBs titer analysis considering it as a continuous variable may have been affected.

In conclusion, our results indicate that BMI may be a factor potentially affecting anti-HBs titer in healthy children. Our findings also revealed a significant correlation between anti-HBs titer and $\mathrm{BMI}$ in children and adolescents, beyond the simple observation that obese people are unlikely to respond to the hepatitis B vaccine.

\section{Conflicts of interest}

No potential conflict of interest relevant to this article was reported.

\section{Acknowledgments}

We are grateful to all subjects and investigators who participated in this study. We also thank the Department of Pediatrics, Bundang CHA Medical Center for help with testing our study participants.

\section{References}

1. Kim YK, Cho SI, Park HS. Obesity as a related factor of poor antibody response to hepatitis B vaccine. Korean J Obes 2003;12:245-51.

2. Simó Miñana J, Gaztambide Ganuza M, Fernández Millán P, Peña Fernández M. Hepatitis B vaccine immunoresponsiveness in adolescents: a revaccination proposal after primary vaccination. Vaccine 1996;14:103-6.

3. Korea Centers for Disease Control \&t Prevention. Seroepidemiology of hepatitis B among preschool children in Korea. Public Health Wkly Rep 2008;1:173-7.

4. Korea Centers for Disease Control \&t Prevention. Achieving the regional goal of the hepatitis B control in Korea. Public Health Wkly Rep 2008;1:273-7.

5. Ahn YO, Kim CY, Bae SH, Chang HG. Changing pattern of HBVrelated diseases in Korea since after HBV vaccination. Clin Mol Hepatol 2010;16:S23-37.

6. Zhao H, Zhou YH. Revaccination against hepatitis B in late teenagers who received vaccination during infancy: Yes or no? Hum Vaccin Immunother 2018;14:456-63.

7. Seo JH. Hepatitis B surface antigen and antibody positive rates of children and adolescents in Jeju. Taehan Kan Hakhoe Chi 2003;9: 304-14.

8. Kim YJ, Li P, Hong JM, Ryu KH, Nam E, Chang MS. A single center analysis of the positivity of hepatitis B antibody after neonatal vaccination program in Korea. J Korean Med Sci 2017;32:810-6. 
9. Van Der Meeren O, Behre U, Crasta P. Immunity to hepatitis B persists in adolescents 15-16 years of age vaccinated in infancy with three doses of hepatitis B vaccine. Vaccine 2016;34:2745-9.

10. Spada E, Romanò L, Tosti ME, Zuccaro O, Paladini S, Chironna M, et al. Hepatitis B immunity in teenagers vaccinated as infants: an Italian 17-year follow-up study. Clin Microbiol Infect 2014;20:0680-6.

11. Jouneghani AS, Chaleshtori MH, Khoshdel A, Kheiri S, Farrokhi E, Khalafian P, et al. Evaluation of response to hepatitis B vaccine in Iranian 6-18-year-old students. J Res Med Sci 2017;22:116.

12. Weber DJ, Rutala WA, Samsa GP, Santimaw JE, Lemon SM. Obesity as a predictor of poor antibody response to hepatitis B plasma vaccine. JAMA 1985;254:3187-9.

13. Kabir A, Pazouki A, Jafari M, Mokhber S, Vaziri M, Alavian SM. Comparing anti-hepatitis B antibody level in iranian obese or overweight with non-obese cases. Iran Biomed J 2017;21:197-202.

14. Fan W, Chen XF, Shen C, Guo ZR, Dong C. Hepatitis B vaccine response in obesity: a meta-analysis. Vaccine 2016;34:4835-41.

15. Korea Centers for Disease Control and Prevention, Division of Health and Nutrition Survey; Korean Pediatric Society, Committee for School Health and Public Health Statistics; Committee for the Development of Growth Standards for Korean Children and Adolescents. 2017 Korean National Growth Charts for children and adolescents. Cheongju (Korea): Korea Centers for Disease Control and Prevention, 2017.

16. The Korean Pediatric Society. Immunization guideline. Seoul: The Korean Pediatric Society, 2018.

17. Young KM, Gray CM, Bekker LG. Is obesity a risk factor for vaccine non-responsiveness? PLoS One 2013;8:e82779.
18. Shaw FE Jr, Guess HA, Roets JM, Mohr FE, Coleman PJ, Mandel EJ, et al. Effect of anatomic injection site, age and smoking on the immune response to hepatitis B vaccination. Vaccine 1989;7:425-30.

19. Poirier MK, Poland GA, Jacobson RM. Parameters potentially affecting interpretation of immunogenicity and efficacy data in vaccine trials: are they adequately reported? Vaccine 1996;14:25-7.

20. Poland GA, Borrud A, Jacobson RM, McDermott K, Wollan PC, Brakke D, et al. Determination of deltoid fat pad thickness. Implications for needle length in adult immunization. JAMA 1997;277:170911.

21. Centers for Disease Control and Prevention (CDC). Intensive-care patients with severe novel influenza A (H1N1) virus infection Michigan, June 2009. MMWR Morb Mortal Wkly Rep 2009;58:74952.

22. Eliakim A, Schwindt C, Zaldivar F, Casali P, Cooper DM. Reduced tetanus antibody titers in overweight children. Autoimmunity 2006; 39:137-41.

23. Banga N, Guss P, Banga A, Rosenman KD. Incidence and variables associated with inadequate antibody titers after pre-exposure rabies vaccination among veterinary medical students. Vaccine 2014;32: 979-83.

24. Karlsson EA, Beck MA. The burden of obesity on infectious disease. Exp Biol Med (Maywood) 2010;235:1412-24.

25. Painter SD, Ovsyannikova IG, Poland GA. The weight of obesity on the human immune response to vaccination. Vaccine 2015;33:44229.

26. Milner JJ, Beck MA. The impact of obesity on the immune response to infection. Proc Nutr Soc 2012;71:298-306. 